\title{
化学分析课程思政具体案例设计及思考
}

宦双燕”, 王玉枝, 蔡炽, 陈增萍, 唐丽娟, 雷春阳, 刘剑波, 李永军 湖南大学化学化工学院, 长沙 410082

摘要: 如何在化学核心课中融入课程思政元素, 形成协同育人效应, 是当前课程建设的一项重要任务。本文介绍了 湖南大学分析化学教学团队在化学分析课程中的课程思政案例设计经验与思考, 围绕化学分析各部分的教学特点, 构建相应的教学案例, 体现课程设计的教学目标和思政育人目标, 同时提出具有普适性的课程思政设计理念。

关键词：课程思政；化学分析；案例设计

中图分类号: G64; O6

\section{The Design and Thinking of the Concrete Case of Ideological and Political Education in Chemical Analysis Course}

Shuangyan Huan ", Yuzhi Wang, Chi Cai, Zengping Chen, Lijuan Tang, Chunyang Lei, Jianbo Liu, Yongjun Li

College of Chemistry and Chemical Engineering, Hunan University, Changsha 410082, China.

\begin{abstract}
How to integrate the ideological and political elements into the core courses of chemistry and form the effect of collaborative education is an important task of the current course construction. This paper introduces the experience and thinking of ideological and political education cases design in the course of chemical analysis from the teaching team of analytical chemistry in Hunan University. Based on the teaching characteristics of chemical analysis, the corresponding teaching cases are constructed to reflect the teaching objectives and ideological and political education objectives. At the same time, the universal design ideas in the ideological and political teaching of the course are put forward.
\end{abstract}

Key Words: Course ideology and politics; Chemical analysis; Cases design

教育是国之大计, 习近平总书记在 2016 年 12 月的全国高校思政工作会议中提出 ${ }^{[1]}$, 要用好课 堂教学这个主渠道，使各类课程与思想政治理论课同向同行，形成协同育人效应。

分析化学课程是湖南大学化学、应用化学专业的学类核心课, 也是化学工程与工艺、生物和医 学等专业的必修课, 以无机化学为先导课程, 为获取物质的化学信息(物质的组成、含量、结构和状 态等信息)提供原理和手段。为实现分析化学课程的协同育人功能, 教学团队经过 “研讨-教学-再研 讨” 反复实践, 构建了系统的课程思政教学案例库, 融知识于案例之中, 同时实现知识传授和思政 教育的双重功能, 创新了分析化学课程课堂教学模式, 激发了学生的学习兴趣和爱国热情。本文将 以分析化学中的 “化学分析” 部分教学为例, 介绍本教学团队在课程教学中的一些方法和举措, 以

收稿: 2020-06-05; 录用: 2020-06-30; 网络发表: 2020-07-29

“通讯作者, Email: syhuan@hnu.edu.cn

基金资助：湖南省普通高等学校课程思政建设研究项目(HNKCSZ-2020-0074) 
促进交流, 逐步提高课程思政的教学效果。

\section{1 绪论部分的课程思政设计}

绪论部分是整个分析化学课程的开篇, 涉及到的宏观性问题较多, 也是最容易融入思政元素的 部分。课程思政的案例设计应建立在学情分析的基础上。本校学习化学分析课程的学生处于大二下 学期, 对于大学课程和专业知识有一定的了解, 课堂积极性不如大一时高。化学分析课程专业性强, 学生虽然学过无机化学和有机化学, 但并不明白它们和分析化学的具体关系, 对分析化学的特点和 重要性不了解, 对身边的分析化学相关案例知其然, 不知其所以然, 因此, 需要培养其理论分析、 批判性思考和开拓创新思维。

\section{1 绪论部分课程思政设计思路与关注点}

就课程而言, 学习一门新课的意义和重要性是学生关注的问题, 也直接决定了学生后续学习的 积极性和主动性。分析化学是一门与人们衣食住行紧密相关的学科, 如何体现分析化学在人们生活、 工农业生产和现代科技发展中的重要作用? 如何引发学生对课程的学习积极性? 在分析化学学科发 展历程中, 有没有中国的学者给我们关键启示? 如何在教学中引入大家广泛关注的社会问题, 从分 析化学的专业角度去深入思考这些问题, 加强学生将所学知识与社会实际问题相结合, 形成自己的 科学观, 也是学习中需要培养的能力。本课程的绪论部分, 结合学情分析和上述这些问题来进行课 程思政案例设计, 将其融入到绪论的知识点内容中, 该思路对于其他课程的绪论部分思政元素融入 具有通用性。

\section{2 绪论部分具体案例教学设计}

案例一: 以 “蔬菜农药残留检测” 为切入点, 说明分析化学在衣食住行、工农业生产和科技强 国中的作用, 体现课程学习的意义和重要性, 弘扬科学观, 总体设计思路如图 1 所示。
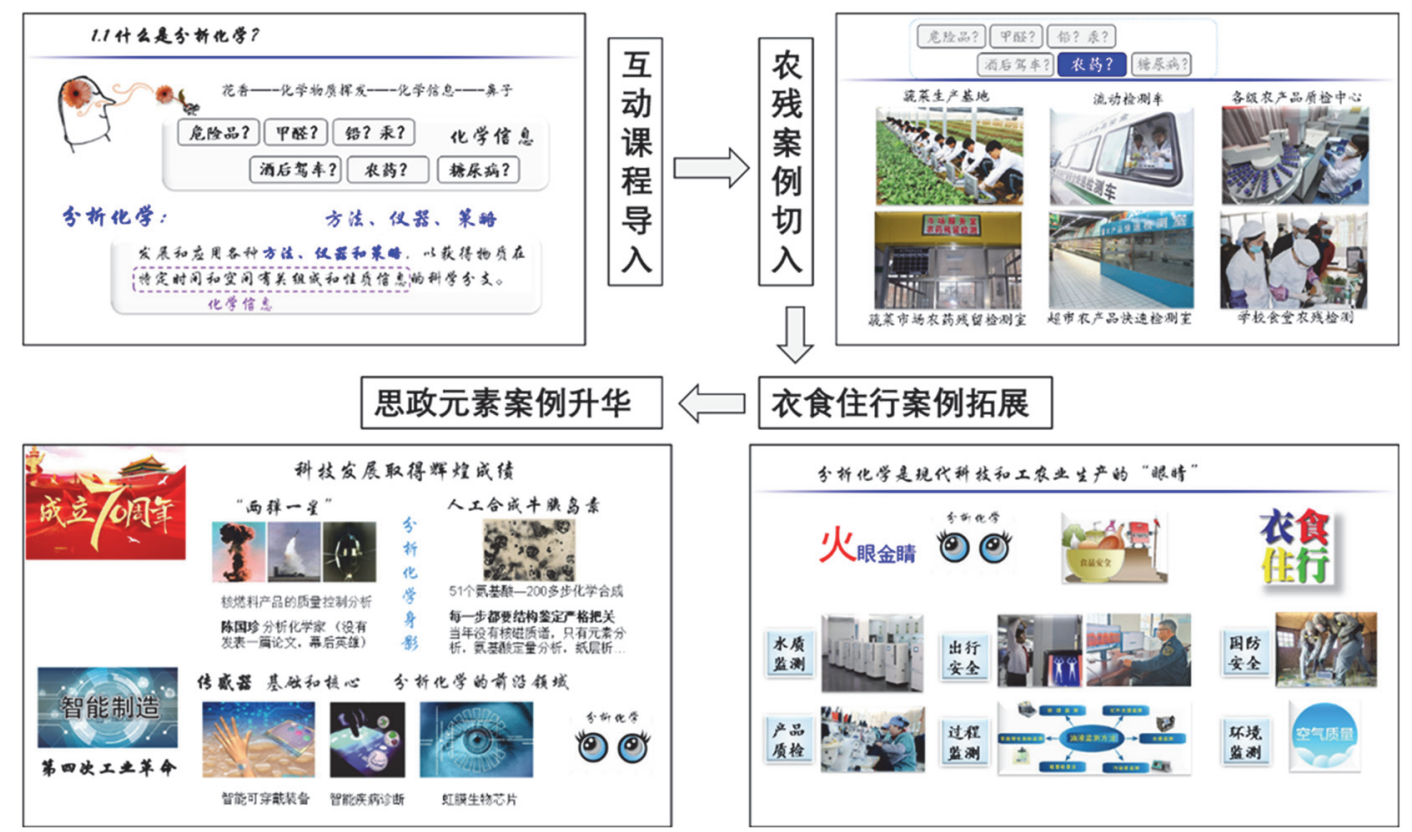

图 1 绪论部分思政案例一的设计思路示意图

在分析化学课程导入部分, 以 “鼻子闻到花香” 这一化学信息的直接获取为切入点, 拓展到 “衣 食住行” 密切相关的问题, 如海关、安检危险品检查, 住房甲醛超标, 化妆品铅录超标, 以及酒驾、 糖尿病、农药残留等化学信息的获取。设计互动问答, 引入分析化学的定义, 同时为后文的案例融 
入做铺垫。

切入: 以 “蔬菜农药残留” 为例 ${ }^{[2]}$, 蔬菜从生产到走上我们的餐桌, 中间经历了多级安全检测, 蔬菜生产基地是重点监测的地方, 各地都设有流动检测车, 农产品有质检中心, 各级蔬菜市场、超 市, 包括食堂都设有农残检测室, 层层把关。分析化学就是检测农药残留的 “眼睛” , 有了分析化 学的 “火眼金睛”, 我们的食品安全才有保障。

拓展: 分析化学其实渗透到了我们衣食住行的方方面面, 水质监测、空气质量监测、工农业生 产过程和产品质量检测、个人健康生化指标检测、医药生产、出行安全、国防安全等等。可以列举 一两个实例，如钢铁冶炼中通过炉前分析控制钢铁质量，工业生产中在线监测控制产品质量。

升华：共和国成立 70 年来，我国的科技发展取得辉煌成绩，大家熟知的如 “两弹一星” “人工 合成结晶牛胰岛素”, 这些成就跟分析化学有没有关系? 分析化学家陈国珍就参与了核燃料产品的 质量控制分析 ${ }^{[3]}$, 因为涉及国家机密, 他没有因此发表一篇相关学术论文, 但他是真正的幕后英雄。 51 个氨基酸经历 200 多步化学合成牛胰岛素 ${ }^{[4]}$, 每一步都要结构鉴定严格把关, 当年没有核磁、质 谱, 只有元素分析、氨基酸定量分析、纸层析等手段分析, 分析化学家是在怎样艰苦的条件下完成 了结构分析的。目前人类社会已进入以 “智能制造” 为代表的第四次工业革命, 智能制造必须要和 各种各样的传感器结合才能实现智能化, 所以传感器的研究是基础和核心, 而化学生物传感正是分 析化学的前沿研究领域, 如智能可穿戴装备、智能疾病诊断系统、虹膜芯片等等。

由此可见, 分析化学是现代科技和工农业生产的 “眼睛” , 一个国家的分析化学水平, 从某种 程度上反映出这个国家的科技水平。依此阐明了学习分析化学课程的意义和重要性。

案例二: 以我国分析化学家高鸿院士的生平事迹, 诠释分析化学工作者应有的特质, 弘扬核心 价值观和科学家精神 ${ }^{[5]}$ 。

通过对我国著名分析化学家高鸿院士生平事迹的深入挖掘, 发现在他身上具有老一辈科学家共 有的一些特质:

（1）报国热忱: 1948 年他放弃美国伊利诺大学的优厚条件, 毅然回国, 在写给未婚妻的信上说: “我知道国内硝烟弥漫, 生活艰苦, 工作条件差, 但我宁愿回国后英雄无用武之地, 也不愿有国不 能回”。他说: “梁园虽好, 并非久留之地”, “我应该为我的祖国和同胞服务, 我的事业在祖国。” 回国后他编写了中国第一部《仪器分析》教科书(1956 年), 开创了我国仪器分析的先河。他一生为 中国的科学事业而奋斗, 汇编出版了《分析化学前沿》。

(2) 严谨求实: 在编写《仪器分析》时, 书中收录的每一个实验, 他都亲自动手做过, 即使条件 非常艰苦, 他也要经过实践验证才编入教材。

(3) 开拓创新：极谱分析研究理论一直被看作是 “高深莫测” 的, 如 “球形电极扩散电流公式”, 极谱学权威学者曾断言这个公式是无法验证的。高鸿经过系列深入的研究, 创造性地提出了 19 个电 化学理论公式, 圆满通过实验验证了 “球形电极扩散电流公式” 的理论, 首创了电滴定分析领域新 技术一一示波滴定分析。

案例三: 以 “镉大米” 为代表的公共安全事件为切入点, 提出研究任务进行互动讨论, 说明分 析化学不仅是数据的提供者, 更是问题的解决者, 加强思辨能力和社会责任感, 总体设计思路如图 2 所示。

分析化学与其他学科相比的一个显著不同之处在于, 它提供的产品是数据。这就延伸出一个问 题: 分析化学工作者是否仅仅是数据的提供者? 这里可以结合公共安全事件等社会广泛关注的问题, 通过具体研究任务和解决方案的互动讨论, 引导学生从分析化学专业的角度去理解, 为什么不仅仅 要提供完善数据, 更要考虑如何解决实际问题。

“镉大米” 事件概要 ${ }^{[6]}: 2013$ 年 2-5 月广东省官方抽检大米及米制品 4600 多批次, 截至 5 月 24 日, 总计公布 155 批次镉含量超标大米的名单。超过半数批次产地为湖南省, 镉含量最高超过国 标 3 倍, 高达 $0.6 \mathrm{mg} \cdot \mathrm{kg}^{-1}$ 。 


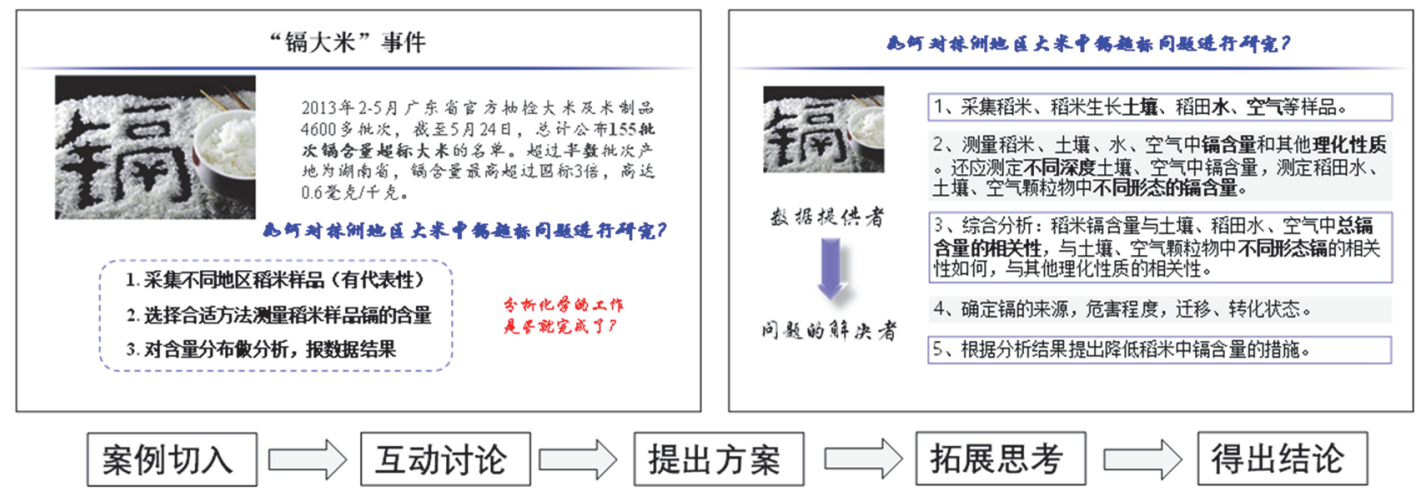

图 2 “镉大米”事件思政案例设计思路

提出思考问题：如何对株洲地区大米中镉超标问题进行研究?

引导学生分别发表观点, 教师进行总结, 由定量分析知识点直接推导出初步研究方案: 1) 采集 不同地区稻米样品(有代表性); 2) 选择合适方法测量稻米样品镉的含量; 3) 对含量分布做分析, 得 到数据结果。

引导学生拓展思考: 得到数据结果后, 分析化学的工作是否就完成了? 没有, 还有哪些可以做 的? 进行生生互动讨论, 在最后提出解决方案:

(1) 采集稻米、稻米生长土壤、稻田水、空气等样品。

(2) 测量稻米、土壤、水、空气中镉含量和其他理化性质。还应测定不同深度土壤、空气中镉含 量, 测定稻田水、土壤、空气颗粒物中不同形态的镉含量。

(3) 综合分析: 稻米镉含量与土壤、稻田水、空气中总镉含量的相关性, 与土壤、空气颗粒物中 不同形态镉的相关性，与其他理化性质的相关性。

(4) 确定镉的来源, 危害程度, 迁移、转化状态。

(5) 根据分析结果提出降低稻米中镉含量的措施。

从该实际问题的讨论可以得出结论: 分析化学工作者不仅是数据的提供者, 更要做问题的解 决者。

\section{2 分析质量保证部分课程思政案例设计}

通过分析质量保证部分的学习, 需要学生掌握误差的基本概念、有效数字及运算规则, 初步学 会实验数据的统计处理方法和提高分析结果准确度的方法, 培养学生正确评价和表达分析结果的能 力。该章节的内容偏数理统计, 在思政融入上可以从马克思主义哲学基本原理和方法论考虑 ${ }^{[7]}$ 。

例如: 在误差概念部分, 系统误差与必然性因素、测量准确度之间的关系, 随机误差与偶然性 因素、测量精密度之间的关系, 以及系统误差和随机误差的客观性, 过失误差的主观性等内容的讲 解能够润物无声地融入马克思主义哲学基本原理与方法论。另外, 在分析结果的统计处理部分, 随 机误差分布的概率分布表现出单峰性、对称性、有界性和抵偿性, 也体现了偶然性和必然性的辩证 统一, 正如恩格斯所说, “在表面上是偶然性在起作用的地方, 这种偶然性始终是受内部的隐蔽的规 律支配的，而问题只是在于发现这些规律”。

在讲授有效数字及运算规则部分内容时, 可以进一步延伸讲解什么是分析化学中 “量” 的概 念 ${ }^{[8]}$ : (1) 取样、读数、用量上的精确; (2) 对成分的准确定量(与厨师炒菜的最大区别); (3) 准确报 结果(保留几位有效数字？方法的误差多大, 偏差多大？)。通过讨论让学生建立对 “量” 的概念的理解。

在分析数据的可靠性检验部分, 学生很难对常规分析数据做可靠性检验的必要性有所理解, 这 里可以将需要分析的数据置换为飞机部件数据, 那么对数据的可靠性分析就变得尤为重要了。案例 
设计时, 可以结合近期热播的电影 “中国机长”, 从挡风玻璃出现裂纹引发的一场空中浩劫展开。

根据汉莎技术公司的数据统计, $85 \%$ 以上的飞机故障是部件可靠性低造成的。什么是部件可靠 性分析 ${ }^{[9]}$ ? 简单地说就是运用适当的数理统计方法对飞机部件数据进行分析, 参照维修标准手册和 世界机群使用数据等标准, 来确定其性能状况和故障趋势, 分析该部件的稳定度和可靠度。从而预 测和判断飞机部件在特定环境及时间内的可靠性状况, 减少空中故障发生率, 为出行安全作保障。

由此可见科学客观地进行数据可靠性检验的重要性。由飞机部件可靠性检验拓展到其他分析数 据的可靠性检验, 如环境污染物的数据、工农业生产中原料和产品的质量数据、食品中的化学添加 成分数据等等, 进一步体现对实际数据进行可靠性检验的重要性和分析中涉及到的社会责任感。

\section{3 滴定分析和重量分析章节课程思政案例设计及其推广的普适性}

\section{1 与历史人物相关的思政案例设计}

在化学分析法导论教学内容中可引入我国近代分析化学家王琎先生提出的科学研究方法 ${ }^{[10]}$ 。王 琎先生在研究中国化学史时发现, 很多化学史料夹杂着政治、迷信等思想, 因此, 他提出以现代科 学手段测定大量数据的基础上, 与历史文献考证相结合的方法来开展化学史的研究。例如: 在《五 铢钱化学成分及古代应用铅、锡、锌、铜考》一文中, 他通过对不同朝代钱币的分析、化验, 并结 合史料, 提出判断钱币年代的科学方法。王琎先生还提倡分析工作要为生产、生活服务, 他撰写的 《南京饮水问题》《江苏凤凰山铁矿的化学分析》《关于浙江黄铁矿的主要成分及含量与其系统分析 方法》等论文都体现了这一点。王琎先生的研究推动了我国化学史的研究和化学分析的发展。

在氧化还原滴定章节中会涉及到能斯特方程, 由这个以人名命名的方程可以延伸到能斯特与哈 伯的历史抉择对比。能斯特不仅提出了能斯特方程, 还建立了热力学第三定律 ${ }^{[11]}$, 发明了能斯特灯、 电钢琴, 和他的导师奥斯特瓦尔德共同提出溶度积等重要概念, 来解释沉淀平衡, 师徒五代相传获 得诺贝尔奖, 也是史上空前的。20世纪初的柏林大学是科学天才的聚集地, 世界学术中心, 如普朗 克、爱因斯坦、海森堡、霍夫曼、德拜; 哲学家黑格尔、叔本华; 社会学家马克思、恩格斯等等, 但德国也诞生了像希特勒这样的法西斯。1930 年之后纳粹党上台, 很多正义的科学家像能斯特、爱 因斯坦等拒绝为纳粹服务, 抗议希特勒暴政而受到纳粹迫害。也有些科学家选择了为纳粹服务, 像 获得 1918 年诺贝尔化学奖的哈伯(犹太裔), 替纳粹研制毒气弹, 成为罪恶的化学武器之父 ${ }^{[12]}$ 。由此 可见，一个科研工作者也要树立正确的价值观和人生观，否则学问可能成为破害人类的武器。

\section{2 公共安全事件与社会责任感相关思政案例设计}

在讲授溶液 $\mathrm{pH}$ 控制的重要性时, 引入 “金龙鱼食用油事件”、“磷化工艺”、日常饮用水的酸碱 性等与生产生活密切相关的问题, 让学生意识到控制溶液酸碱性的重要意义。还可进一步结合实验 室 “三废”处理问题，加强环保意识。

在讲授酸碱滴定法定量分析的氨含量测定时, 可介绍凯氏定氮法, 结合 2008 年席卷全国的三聚 氧胺 “毒奶粉事件” [13]，通过直观的案件回放视觉 “冲击”, 让学生认识到 “蛋白质定量分析方法” 本身的缺陷, 以及熟悉该方法的相关人员的职业道德的缺失所产生的严重后果。进一步体现分析化 学必须发展精准的检测技术, 为食品安全保驾护航。而作为未来的化学或分析化学工作者的学生们, 更是要遵守职业道德，肩负起社会责任感。

\section{3 倡导健康生活方式思政案例设计}

在讲授氧化还原反应的方向和程度时, 联系到氧化还原反应也是人体内最基本的化学反应, 人 体在氧化-抗氧化之间维持一个机体的平衡。众所周知, 通过浓度、酸度、产生沉淀等外因可以改变 一个氧化还原反应的方向。同样的, 人体遭受有害刺激的时候, 也会产生大量活性氧、活性氮自由 基, 氧化-抗氧化失去平衡, 就会产生氧化应激, 严重的导致衰老、疾病等。哪些因素会打破平衡? 鳌夜、抽烟、酗酒、暴饮暴食、过度劳累等等不健康的生活方式, 就会让平衡向氧化方向移动, 加 大疾病风险, 因此需要提倡健康的生活方式, 让机体维持一个稳定的氧化-还原平衡状态。 


\section{4 理论联系实际相关案例设计}

化学分析部分的讲授侧重基本方法、原理和计算, 可以针对性地设计相关方法的实际应用案 例 ${ }^{[14,15]}$, 如饮用水中所含离子的定量分析, 具体包括总硬度、总碱量、溶解性总固体、化学需氧量 的分析, 以及水中钙、镁、铁、氯、氟等离子的定量分析。将所学滴定分析和重量分析的方法原理 应用于实际问题的解决。

其次, 在化学分析的方法原理中较少涉及到实际样品的预处理和国家标准方法的介绍, 可有针 对性地设计食品样品、环境样品、生物样品、材料样品的预处理和国家标准分析方法介绍, 拓展学 生对实际样品分析的理解和综合应用能力。

另外, 沉淀的溶解度相关原理与工业中的污水处理密切相关, 如含氟工业废水处理、氢氧化物 沉淀处理工业废水中的 $\mathrm{Fe}^{3+}$ 和 $\mathrm{Al}^{3+}$ 的酸度控制、以钡盐为沉淀剂处理含铬污水中的碳酸根等等。可 设计相关案例加强学生运用所学知识分析解决实际问题的能力。

\section{5 倡导绿色化学相关案例设计}

在配位滴定法用于重金属离子检测的应用中, 以 “铅中毒” “铝中毒” 等食品和环境安全问题作 为切入点, 引导学生思考汽车尾气排放、易拉罐包装等个人日常生活小问题与人类健康大问题之间 的联系，启发思考降低重金属污染，倡导绿色化学的意识。

在沉淀滴定法中介绍采用微量滴定管进行摩尔法的滴定分析实验, 微量滴定的滴定体积可控制 在 3 毫升以内, 大大减少污染物排放, 体现了绿色化学理念。

\section{4 教师在开展课程思政教育中的几点思考}

教师为人师表, 在课程思政教学中, 更需要以身作则, 不断提高自身师德修养, 以德立身、以 德立学、以德施教、以德育德, 通过言传身教进行隐性渗透式课程思政教育。

一门课程的思政建设需要教学团队成员的共同参与, 首先在思想意识上形成专业培养与立德树 人并重的共识, 其次在思政元素和思政案例设计上的共同参与, 然后是课程教学中的主动实施。只 有教学团队的教师们共同参与课程思政建设，才能对更多的学生产生积极影响。

而在课程思政案例融入时, 也需要把握一个度的问题, 专业课程毕竟不是思政课, 思政元素不 能全盘灌溉式进行, 避免长篇大论的宣讲, 引起学生的反感与抵触。本文中虽然介绍了较为详细的 化学分析课程思政案例设计, 但只是作为一个参考性案例库, 具体的实施还需要教师结合实际教学 情况进行选择和取舍, 执行中要注意思政案例与专业知识的融合度, 把握好思政融入的分寸和比例, 做到恰到好处，才能起到同向同行的育人效果。

\section{参 考 文 献}

[1] 新华社. 习近平: 把思想政治工作贯穿教育教学全过程. (2016-12-08) [2020-03-26].

http://www.xinhuanet.com//politics/2016-12/08/c_1120082577.htm

[2] 许艇, 李季. 大学化学, 2003, 18 (6), 27.

[3] 郭保章. 化学通报, 1996, No. 12, 54.

[4] 叶蕴华. 生命科学, 2015, 27 (6), 648.

[5] 《分析化学》编委会, 《分析化学》编辑部. 分析化学, 2008, 36 (6), 插 1.

[6] 曾艺坤, 练樹斌, 刘健炜, 黄思满, 晏宇翔, 戴福婷. 中外食品工业(下半月), 2015, No. 3, 59.

[7] 李爱峰, 王术皓, 贾丽萍, 韩昭君. 大学化学, 2018, 33 (6), 53. 
[8] 朱霞萍, 胡晓荣, 孙永华, 周莉. 大学教育, 2016, No. 6, 104 .

[9] 孙璐. 大科技·科技天地, 2010, No. 10, 106.

[10] 王锦光. 中国科技史杂志, 1988, No. 4, 92 .

[11] 赵玉杰, 杨谦, 王洪见. 大学物理, 2014, 33 (1), 51.

[12] 褚廷夫, 周建娥, 王世增. 化学教学, 1999, No. 11, 17.

[13] 王丹, 赵美萍. 大学化学, 2009, 24 (1), 9.

[14] 王玉枝, 旷亚非, 宦双燕, 郭慢丽, 王青. 化工高等教育, 2010, 112 (2), 75.

[15] 王玉枝. 大学化学, 2017, 32 (5), 65. 